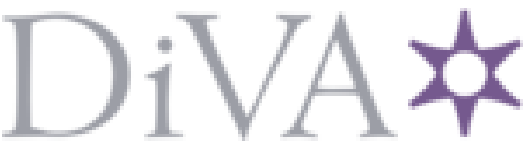

http://www.diva-portal.org

Postprint

This is the accepted version of a paper presented at Solid-State Sensors, Actuators and Microsystems (TRANSDUCERS), 2017 19th International Conference on.

Citation for the original published paper:

Schröder, S. (2017)

A LOW-COST NITRIC OXIDE GAS SENSOR BASED ON BONDED GOLD WIRES.

In:

N.B. When citing this work, cite the original published paper.

Permanent link to this version:

http://urn.kb.se/resolve?urn=urn:nbn:se:kth:diva-214452 


\title{
A LOW-COST NITRIC OXIDE GAS SENSOR BASED ON BONDED GOLD WIRES
}

\author{
Stephan Schröder ${ }^{1,2}$, Hithesh K. Gatty ${ }^{1}$, Göran Stemme ${ }^{1}$, Niclas Roxhed ${ }^{1}$ and Frank Niklaus ${ }^{1}$ \\ ${ }^{1}$ Department of Micro and Nanosystems, KTH Royal Institute of Technology, Stockholm, SWEDEN \\ ${ }^{2}$ SenseAir AB, Delsbo, SWEDEN
}

\begin{abstract}
In this paper we report of a novel and very simple fabrication method for realizing amperometric gas sensors using conventional wire bonding technology. Working and counter electrodes are made of 360 vertically standing bond wires, entirely manufactured by a fully automated, standard wire bonding tool. Our process enables standing bond wires with a length of $1.24 \mathrm{~mm}$, resulting in an extremely high aspect-ratio of 50, thus effectively increasing the surface area of the working electrode. All gas sensor electrodes are embedded in a polymer-based, solid electrolyte. Therefore, laborious handling of liquid electrolytes can be avoided. Here, we report of a nitric oxide (NO) gas sensor that is capable of detecting NO gas concentrations down to the single-digit ppm range. The proposed approach demonstrates the feasibility towards a scalable and entire back-end fabrication concept for lowcost NO gas sensors.
\end{abstract}

\section{KEYWORDS}

Amperometric gas sensor, nitric oxide gas sensing, NO gas sensor, wire bonding, vertical standing bond wires.

\section{INTRODUCTION}

Electrochemical gas sensing is becoming increasingly important and plays a key role in many industries. Especially prominent are gas sensors based on the amperometric operating principle, which are crucial devices in various applications for example in the health care sector, i.e. in hygiene and medical applications, for environmental monitoring of greenhouse gases and in heavy industry applications such as gas stack and combustion monitoring [1]. Amperometric gas sensors (AGS) allow to accurately sense and quantify specific trace gases, in the low ppm and ppb range, such as hydrogen $\left(\mathrm{H}_{2}\right)$, carbon monoxide $(\mathrm{CO})$, sulfur dioxide $\left(\mathrm{SO}_{2}\right)$ and nitric oxide (NO). $\mathrm{NO}$, for example, is generated, as non-negligible byproduct, in combustion processes of fossil fuels and in the production of fertilizers, resulting subsequently in the formation of $\mathrm{NO}_{2}$ which is considered as highly effective greenhouse gas $[3,4,5]$. NO is a toxic gas and excessive exposure is classified as harmful to health and thus, the legal limit of exposure to NO have been set at work places to $25 \mathrm{ppm}$ in the US [2]. Thus, there is a need for miniaturized and portable NO gas sensors in various settings such as at work places with hazardous environments. Commercially available gas sensing instruments are often bulky, while available MEMS-based NO gas sensors can offer a promising alternative, for example previously reported miniaturized amperometric NO gas sensors with a fast response time [6]. However, MEMS-based NO gas sensors often rely on complex fabrication methods and (a)

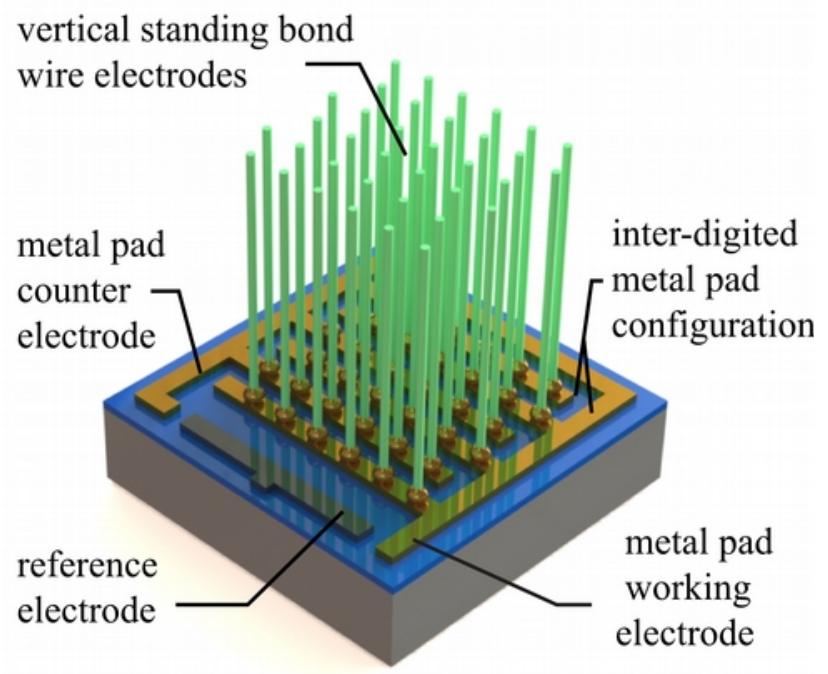

(b)

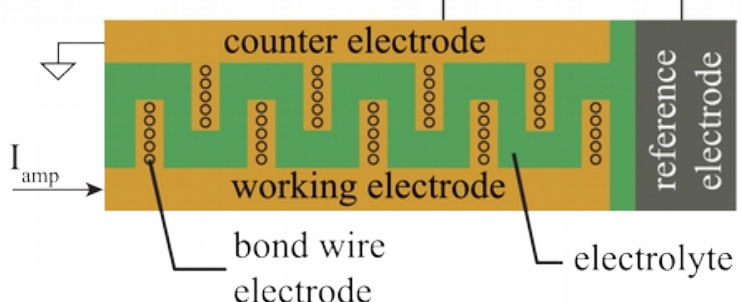

Figure 1:Illustration of the NO gas sensor and its working principle. (a) Counter and working electrodes are defined by vertically standing bond wires on top of the inter-digited metal pads. The reference electrode is an in-plane defined silver pad. The entire device is covered by a polymer-based solid electrolyte (indicated in green only on the vertical standing bond wire electrodes). (b) NO gas is oxidized at the working electrode while a potentiostatic circuit maintains a constant potential between the reference and working electrodes of $0.95 \mathrm{~V}$. The current $I_{\text {amp }}$ is linearly proportional to the NO gas concentration.

expensive substrate materials, such as silicon-on-insulator (SOI) substrates [7]. Instead, this paper reports of a novel, scalable and low-cost manufacturing method for realizing miniaturized $(10 \mathrm{~mm} \times 10 \mathrm{~mm} \times 1.4 \mathrm{~mm})$ amperometric NO gas sensors that are fabricated using conventional wire bonding technology. Wire bonding is a very mature packaging technology for establishing electrical interconnections between IC dies and its package. This technology is a fully automated manufacturing method offering very-high throughput and high placement accuracy of bond wires of below $3 \mu \mathrm{m}$. Consequently, wire bonding is a promising and attractive technology for cost-efficient fabrication. Wire bonding has been recently proposed for several innovative packaging and fabrication 
approaches for MEMS devices [8, 9, 10]. In this work conventional gold ball wire bonding is proposed for fabricating working and counter electrodes of the NO gas sensor by realizing vertically standing bond wires with a length of $1.24 \mathrm{~mm}$ that are placed on metal electrode pads and thus, effectively increasing the surface area of the electrodes. No modification of the standard wire bonding tool is required for the proposed fabrication process.

\section{SENSOR DESIGN AND FABRICATION}

Our sensor design is based on a conventional three electrode configuration, consisting of working, counter and reference electrodes. Figure 1a depicts a 3D model of the proposed NO gas sensor. Working and counter electrode pads are defined in-plane and patterned in an inter-digited shape on top of the substrate. The working and counter electrodes are fabricated by placing vertically standing bond wires on top of the electrode pads, thereby forming a dense pillar forest. Vertically standing bond wires on top of the counter electrode ensure an uniform current density. The reference electrode is defined in-plane and located next to the working and counter electrode pads. The entire device is covered by a thin layer of a solid polymer-based electrolyte, type Nafion and thus all electrodes are in electrical contact and an electrochemical cell is established. For clarity reasons the polymer-based electrolyte, highlighted in green, is only indicated on the vertically standing bond wire electrodes in figure 1a. The usage of Nafion as a solid polymer electrolyte allows a simplified sensor design and laborious electrolyte handing is per se avoided. The concept of vertically standing bond wire electrodes permit a good interface between the working electrode to the ambient gas environment. In figure $1 \mathrm{~b}$ a simplified layout of the NO gas sensor is illustrated. A potentiostatic circuit maintains a constant potential between the working and reference electrodes of $0.95 \mathrm{~V}$. NO gas molecules in the ambient atmosphere diffuse through the solid electrolyte to the surface of the vertical standing bond wire working electrode and being there oxidized as part of a redoxreaction within the electrochemical cell of the NO gas sensor. The resulting current flow $\left(\mathrm{I}_{\mathrm{amp}}\right)$ at the working electrode pad is directly proportional to the redox-reaction and therefore is a measure of the NO gas concentration. Our proposed sensor concept provides the prospect of very low-cost NO gas sensors as substrate fabrication can potentially be realized using conventional printed circuit board manufacturing techniques, and consequently costly MEMS substrate manufacturing processing can be avoided. Furthermore the usage of wire bonding technology for placing the vertically standing bond wires enables the cost-efficient fabrication of the electrodes. In this proof-of-concept we are focusing on the novel fabrication approach of the standing bond wire electrodes and for simplicity a silicon substrate is utilized and the metal electrode pads are manufactured using conventional MEMS processing technology.

\section{Substrate Fabrication}

A single-side polished silicon wafer with a thickness of $500 \mu \mathrm{m}$ and a diameter of $100 \mathrm{~mm}$ is oxidized to grow (a) $\mathrm{SiO}_{2}$ (hardmask)

(silicon substrate)
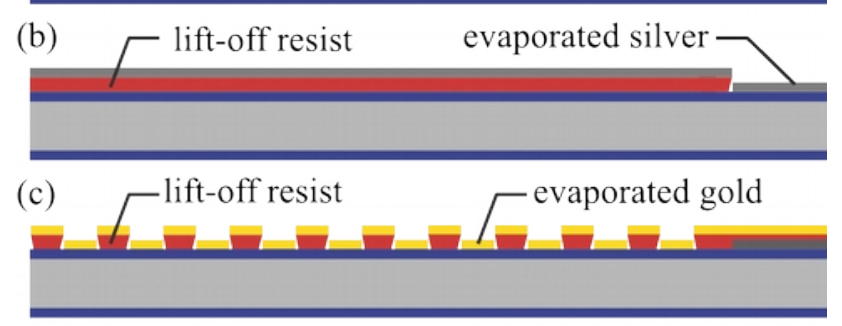

(d) $\Gamma$ inter-digited working \& counter electrode pads

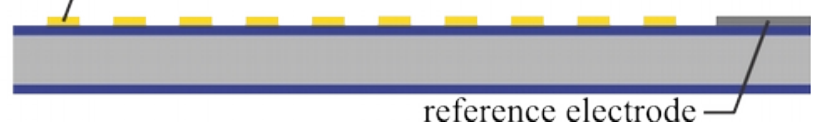

Figure 2: Illustration of the process flow for the substrate fabrication. (a) Thermal oxidation of a single-side polished silicon wafer to grow a $1 \mu \mathrm{m}$ thick insulation layer of silicon dioxide. (b) Formation of the reference electrode by evaporation of $500 \mathrm{~nm}$ of silver in combination with a lift-off process. (c) Lithographic patterning and evaporation of $10 \mathrm{~nm}$ of titanium and $200 \mathrm{~nm}$ of gold. (d) Finalizing the inter-digited metal pad formation of the working and counter electrode using liftoff process.

a $1 \mu \mathrm{m}$ thick silicon dioxide $\left(\mathrm{SiO}_{2}\right)$ layer for electrically insulating the substrate from the electrode pads (figure 2a). The reference electrode is patterned using lithography and evaporation of $500 \mathrm{~nm}$ of silver in combination with a lift-off process (figure 2b). Next, lithographic patterning defines the layout of the interdigited in-plane metal pads of the working and counter electrodes, simultaneously. Evaporation of $10 \mathrm{~nm}$ of titanium as an adhesion layer and $200 \mathrm{~nm}$ of gold follows (figure 2c). A conventional lift-off process generates both electrodes and finalizes the substrate fabrication (figure 2d).

\section{Formation of the Vertical Bond Wire Electrodes}

Chips with a dimension of $10 \mathrm{~mm}$ by $10 \mathrm{~mm}$ are diced and placed on the heater plate of the wire bonder which is set to a temperature of $60{ }^{\circ} \mathrm{C}$. In this work a fully automated, standard wire bonder, type ESEC 3100 plus, BESI, Switzerland, is utilized for the formation of the vertical bond wire electrodes. No modification of the wire bond tool is required. The vertical bond wire formation is performed using gold wire with a diameter of $25 \mu \mathrm{m}$ and a wire bond capillary for deep access packaging applications type SBNS-35-DP-C-1/16-XL, SPT, Switerland is utilized. First, a free air ball (FAB) with a diameter of $75 \mu \mathrm{m}$ is formed by an electronic flame off (EFO) using current of $40 \mathrm{~mA}$ and a firing time of $1.23 \mathrm{~ms}$. The wire bond capillary moves to the ball bond position which is located on the working electrode pad. A conventional ball bond is performed using a bond force of $500 \mathrm{mN}$, an ultrasonic energy of $30 \%$ and a bond time of $12 \mathrm{~ms}$ (figure $3 \mathrm{a}$ ). The predefined looping type, i.e. the trajectory that the wire bond capillary performs to render the final shape of the bond wire, for flat loop bond wires 
(a)

(b)

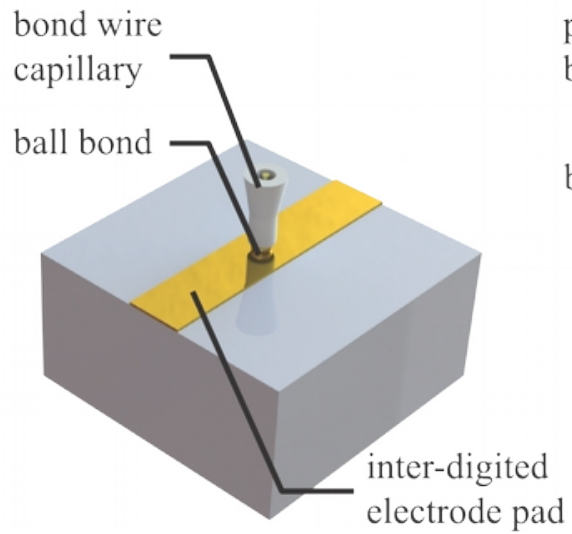

(c)

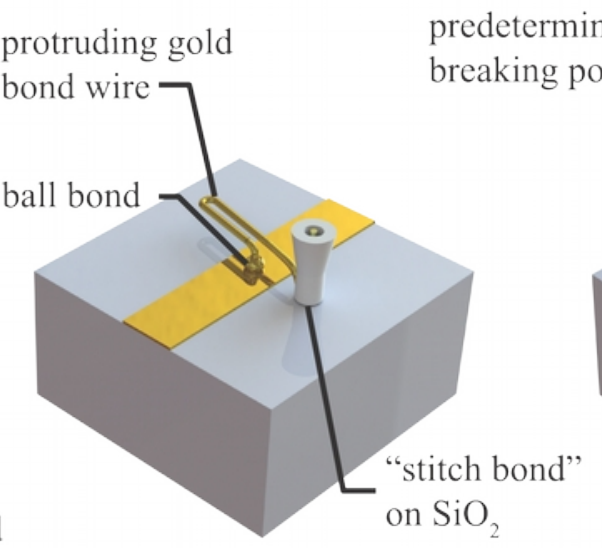

bond wire rupture

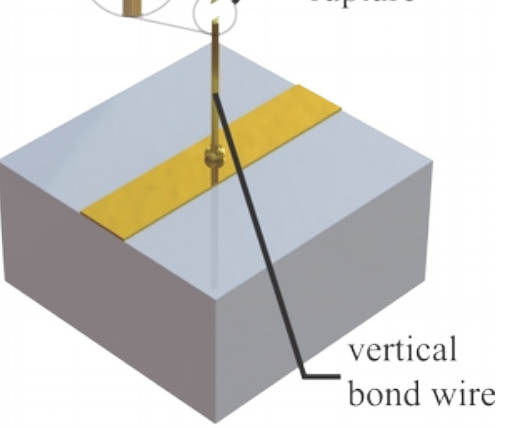

Figure 3: Illustration of the vertically standing bond wire fabrication.(a) A conventional ball bond is performed on the inter-digited electrode metal pad. (b) The bond wire capillary performs the trajectory for the formation of flat loop bond wires. The combination of a loop height of $600 \mu \mathrm{m}$ and the distance of $150 \mu \mathrm{m}$ between the ball bond and stitch bond position results in a protruding and folded bond wire. The performed "stitch bond" without establishing a micro welding connection on the $\mathrm{SiO}_{2}$ layer generates a predetermined breaking point in the bond wire. (c) Vertical lifting up of the wire bond capillary straightens the wire. At the tail generation height the wire clamp closes and the wire is torn off at the predetermined breaking point.

has been chosen in combination with a loop height of $600 \mu \mathrm{m}$. The stitch bond position is located on the $\mathrm{SiO}_{2}$ layer of the sensor substrate next to the working electrode pad. The distance of $150 \mu \mathrm{m}$ between the ball bond and the stitch bond positions is extremely short with respect to the loop height. As a consequence of the combination of the extended loop height and the short distance between both bond positions, a protruding bond wire is generated and folded close to the substrate, while the wire bond capillary moves to the stitch bond position (figure $3 \mathrm{~b}$ ). The length of the vertically standing bond wire is defined by about the double of the defined loop height for the flat loop wire type, resulting in an out-of-plane length of the vertically standing bond wires of $1.24 \mathrm{~mm}$ with an aspect ratio of about 50 . To form a breaking point on the bond wire a "stitch bond" is performed using $200 \mathrm{mN}$ and a bonding time of $10 \mathrm{~ms}$ (figure $3 \mathrm{~b}$ ). However no micro-welding connection between the gold bond wire

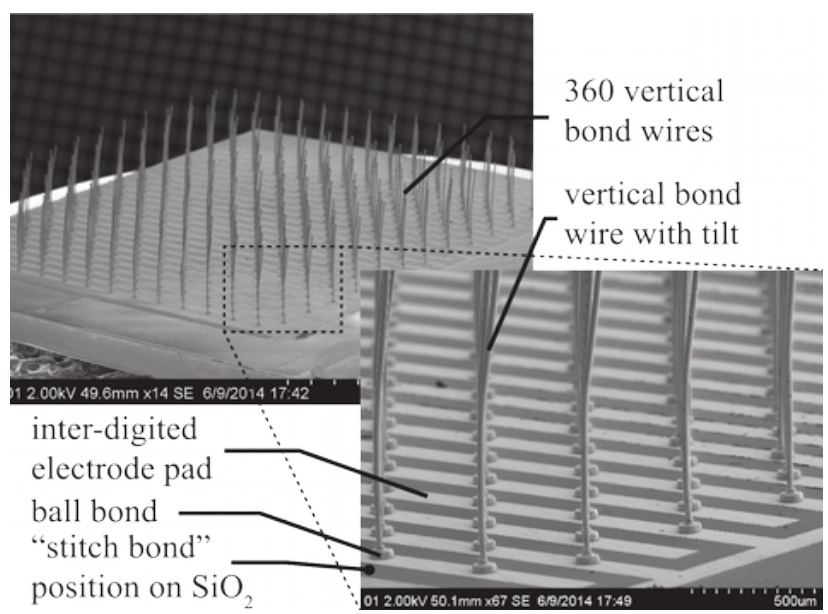

Figure 4: Image of the NO gas sensor chip after placing 360 vertical bond wires to generate the the working and counter electrode. The close-up illustrates details of the stitch bond position and shows a consistent tilt of the vertical bond wires. and the $\mathrm{SiO}_{2}$ surface is established. Instead the predetermined breaking point is generated. Next, the wire bond capillary moves upwards and straightens the bond wire. At the height for generating a wire tail, the wire clamp closes while the wire bond capillary moves further upwards. The bond wire breaks at the predetermined breaking point and the formation of the vertically standing bond wire is finalized (figure 3c). The time for forming one vertically standing bond wire amounts to $320 \mathrm{~ms}$ and is repeated for each wire position on the working and counter electrode pads until a total number of 360 vertical standing bond wires with a pitch $500 \mu \mathrm{m}$ are fabricated.

In a final step, the fabricated device is dip coated in a $5 \%$ Nafion solution (SE-5112, DuPont, USA) and dried in air for approximately $10 \mathrm{~min}$.

\section{FABRICATION RESULTS AND SENSOR CHARACTERIZATION}

Fabricated vertically standing bond wires have been inspected using SEM. Figure 4 shows the NO gas sensor substrate after establishing 360 vertical bond wires on the working and counter electrode pads. The close-up in figure 4 shows details of the vertical bond wire electrodes. All vertical bond wires showing a consistent tilt to on side that ranges between $180 \mu \mathrm{m}$ and $147 \mu \mathrm{m}$. The observed tilt is caused by stretching the vertical bond wire, as the bond wire capillary moves upwards at the stitch bond position, i.e. $150 \mu \mathrm{m}$ laterally shifted away from the ball bond position.

\section{NO sensor characterization}

Characterization of the fabricated device is performed by exposing the NO gas sensor to a controlled atmosphere in a custom-made setup. The measurement setup is schematically depicted in figure 5 . The NO sensor is connected to a potentiostat (DY2011, Digi-ivy, USA) and a voltage between working and reference electrodes is maintained at a fixed value of $0.95 \mathrm{~V}$. The current at the 


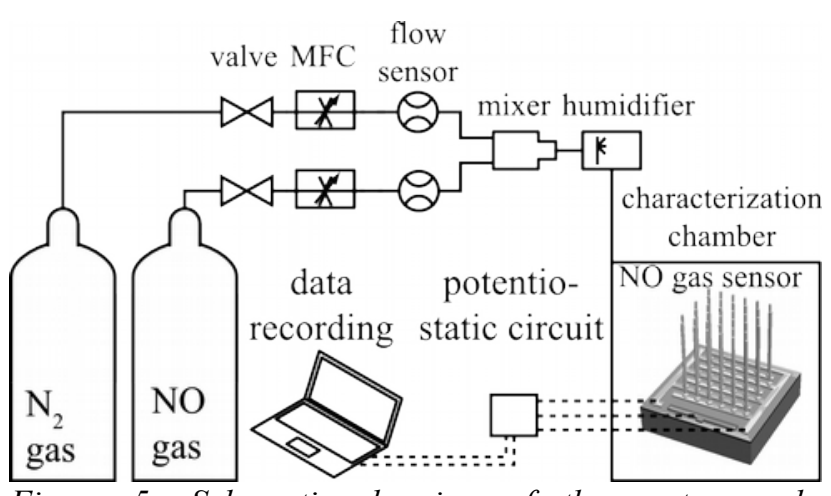

Figure 5: Schematic drawing of the custom-made measurement setup for characterizing the NO gas sensor. $\mathrm{N}_{2}$ and NO gas bottles are connected to a mass flow controller, a mixer and humidifier to generate a defined atmosphere in the measurement chamber. The NO sensor is connected to potentiostatic circuit and the current flow is recorded, while the gas concentration of NO is varied.

working electrode $\left(\mathrm{I}_{\mathrm{amp}}\right)$ is recorded using the potentiostat. A nitrogen $\left(\mathrm{N}_{2}\right)$ gas bottle and a gas containing $90 \mathrm{ppm}$ $\mathrm{NO}$ in $\mathrm{N}_{2}$ are connected to a mass flow controller (MFC) (F201CV, Bronkhorst EL-flow, Netherland) and to two flow sensors (AWM5102, Honeywell, USA). Humidity is maintained at $37 \% \mathrm{RH}$ using a humidifier. The measurement set-up is controlled using a computer with a labview interface. Varying of the NO gas concentration is performed by utilizing a mixer. In order to maintain a constant flow of gas with a specific composition, the flow rates of $\mathrm{NO}$ and $\mathrm{N}_{2}$ are recorded using both flow sensors. The experiment was performed by switching the gases from $\mathrm{N}_{2}$ to $\mathrm{NO}$ and back to $\mathrm{N}_{2}$. The gas flow to the sensor was maintained at constant flow of $600 \mathrm{ml} / \mathrm{min}$. Figure 6 depicts the working electrode current for different NO gas concentrations. The NO gas sensor shows a response time of about $19 \mathrm{~s}$, which is attributed to the thin layer of Nafion coating that allows a short diffusion path of NO gas to the working electrode surface.

\section{CONCLUSION}

We have shown a novel and simple fabrication method for NO sensors using conventional wire bonding technology. Working and counter electrodes are formed by 360 vertical standing bond wires with a height of $1.24 \mathrm{~mm}$. The fabricated $\mathrm{NO}$ gas sensor is capable of detecting NO gas concentrations in the single-digit ppm range and shows low background currents. The presented

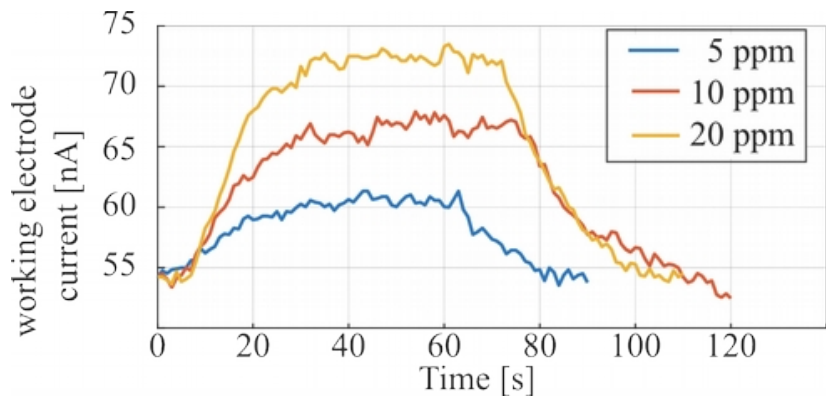

Figure 6: Plot of the current response of NO concentration of $5 \mathrm{ppm}, 10 \mathrm{ppm}$ and $20 \mathrm{ppm}$. The sensor has a response time of approximately $19 \mathrm{~s}$ and shows low background currents if the sensor is not exposed to NO. fabrication concept offers the prospect of an entirely backend fabrication process of very low-cost NO gas sensors that have the potential to be utilized as an indoor air quality measurement sensor for example at work places with hazardous environments.

\section{ACKNOWLEDGEMENTS}

Support from the European Commission through the ERC Starting Grant (M\&M's, 277879) is gratefully acknowledged.

\section{REFERENCES}

[1] Stetter, Joseph R., and Jing Li. "Amperometric gas sensors a review." Chemical reviews 108.2 (2008): 352-366.

[2] https://www.osha.gov/dts/chemicalsampling/data/CH 256700

[3] Crutzen, Paul J. "The influence of nitrogen oxides on the atmospheric ozone content." Quarterly Journal of the Royal Meteorological Society 96.408 (1970): 320325.

[4] Twigg, Martyn V. "Progress and future challenges in controlling automotive exhaust gas emissions." Applied Catalysis B: Environmental 70.1 (2007): 215.

[5] Wood, Sam, and Annette Cowie. "A review of greenhouse gas emission factors for fertiliser production." IEA bioenergy task. Vol. 38. No. 1. 2004.

[6] Gatty, Hithesh K., et al. "An amperometric nitric oxide sensor with fast response and ppb-level concentration detection relevant to asthma monitoring." Sensors and Actuators B: Chemical 209 (2015): 639-644.

[7] Gatty, Hithesh K., Göran Stemme, and Niclas Roxhed. "A wafer-level liquid cavity integrated amperometric gas sensor with ppb-level nitric oxide gas sensitivity." Journal of Micromechanics and Microengineering 25.10 (2015): 105013.

[8] Mohammed, Ilyas. "Fine pitch copper interconnects for next generation package-on-package (pop)." International Symposium on Microelectronics. Vol. 2012. No. 1. IMAPS, 2012.

[9] Schröder, Stephan, et al. "Stress-minimized packaging of inertial sensors by double-sided bond wire attachment." Journal of Microelectromechanical Systems 24.4 (2015): 781-789.

[10] Schröder, Stephan, et al. "Fabrication of an infrared emitter using a generic integration platform based on wire bonding." Journal of Micromechanics and Microengineering 26.11 (2016): 115010.

\section{CONTACT}

S. Schröder, tel: +46702555337; stephan.schroder@ee.kth.se; stephan.schroder@senseair.se.

H. K. Gatty, tel: +46760790758; hithesh@kth.se. 